\title{
Application of Topical Sucralfate and Topical Platelet-Rich Plasma Improves Wound Healing in Diabetic Ulcer Rats Wound Model
}

\author{
Renni Yuniati $\mathbb{D}^{\prime}$ \\ Innelya Innelya $\mathbb{D}^{2}$ \\ Arti Rachmawati $\mathbb{D}^{2}$ \\ Harold Jefferson Matthew \\ Charlex (iD $^{2}$ \\ Alfi Rahmatika (iD ${ }^{2}$ \\ Matthew Brian Khrisna ${ }^{1}$ \\ Farmaditya EP Mundhofir (1D ${ }^{3}$ \\ K Heri Nugroho Hario Seno (iD) 4 \\ Tri Nur Kristina $\mathbb{D D}^{5}$ \\ 'Dermatology and Venereology \\ Department, Faculty of Medicine, \\ Diponegoro University, Semarang, \\ Indonesia; 'Undergraduate Student, \\ Faculty of Medicine, Diponegoro \\ University, Semarang, Indonesia; \\ ${ }^{3}$ Histology Department, Faculty of \\ Medicine, Diponegoro University, \\ Semarang, Indonesia; ${ }^{4}$ Internal Medicine \\ Department, Faculty of Medicine, \\ Diponegoro University, Semarang, \\ Indonesia; ${ }^{5}$ Microbiology Department, \\ Faculty of Medicine, Diponegoro \\ University, Semarang, Indonesia
}

Purpose: One of the most serious and devastating complications of diabetes mellitus is diabetic ulcers. They are difficult to treat and often result in limb loss. Topical sucralfate and platelet-rich plasma have the potential to improve the healing outcomes of chronic ulcers, including diabetic ulcers. This research aims to determine the effectiveness of sucralfate and platelet-rich plasma therapy for the improvement of diabetic ulcer wound healing.

Patients and Methods: Ninety Wistar rats were used in this study and were classified into five groups. Four of the five groups were diabetic induced and were treated with topical sucralfate only, platelet-rich plasma only, combination of topical sucralfate and platelet-rich plasma, and diabetic control group which received standard therapy only. The non-diabetic control group did not receive any therapy. We observed macrophage amount, platelet-derived growth factor, vascular endothelial growth factor, and hypoxia-inducible factor as a biomarker. Rats were terminated after 7 th and 14 th days and were subjected to immunohistochemistry staining and examination.

Results: We found that topical sucralfate and platelet-rich plasma increase macrophage levels, vascular endothelial growth factor expression and platelet-derived growth factor expression in diabetic wound cells. We also found a reduction in hypoxia inducible factor$1 \alpha$ expression. Combination of topical sucralfate and platelet-rich plasma for 14 days gave the most significant improvement in terms of wound healing compared to topical sucralfate or platelet-rich plasma alone.

Conclusion: The combination of topical sucralfate and platelet-rich plasma therapy results in the best improvement in diabetic ulcer wound healing compared to sucralfate or plateletrich plasma monotherapy or conventional wound healing therapy.

Keywords: hypoxia inducible factor, VEGF, PDGF, macrophage

\section{Introduction}

One of the most devastating complications of diabetes mellitus is diabetic ulcers. Not only because these ulcers heal slowly, these ulcers may also cause disability and even results in limb loss. ${ }^{1}$ A diabetic ulcer is a chronic wound usually found in the soles of the diabetic patient's feet. The occurrence of diabetic ulcers is mostly associated with neuropathy and vasculopathy in the form of the peripheral arterial disease which happens in the lower limb of the diabetic patient. ${ }^{2}$ Around $2 \%$ to $5 \%$ of all population in the world suffers from diabetic ulcers. ${ }^{3}$ The hyperglycemic state in diabetics causes molecular and physiological changes that cause diabetic ulcers to become difficult to heal, increasing its risk to secondary infection and potentially
Correspondence: Renni Yuniati Dermatology and Venereology Department, Faculty of Medicine, Diponegoro University, Semarang, Indonesia

Tel +628122874008

Email renniyuniati@yahoo.com 
causing limb amputation if it is not treated properly. ${ }^{4}$ Diabetic ulcers account for nearly $90 \%$ of all lower limb amputation cases, with a reported mortality rate per year of $5.5 \%$ due to diabetic ulcers. ${ }^{5,6}$

The main aim of diabetic ulcer therapy is to prevent extensive damage and secondary infection of diabetic ulcers, thereby minimizing the risk of further damage or even limb amputation. A thorough therapy for diabetic ulcers includes wound debridement, wound dressing, revascularization procedures, infection management, and ulcer off-loading. ${ }^{2}$ One of the primary problems in diabetic ulcer wound healing is the difficulty of triggering the regeneration process for wound healing, although the wounds have been treated in a good manner. Even when the patient is treated with gold-standard therapy, the healing of diabetic ulcers still needs 6 months average healing time with a healing rate of $30 \%$. However, $14 \%$ of patients to be amputated and $12.8 \%$ mortality rate adverse outcomes still exist even with gold-standard therapy. ${ }^{7}$ Therefore, we can conclude that the therapy for diabetic ulcers took a long period, yet not satisfactory enough, and is not cost-effective. ${ }^{8,9}$

Sucralfate is a cytoprotective agent that is mainly used for the treatment of gastrointestinal tract ulcers, including gastric ulcers, duodenal ulcers, and gastritis. Newer researches show that sucralfate can also be used topically in open wounds and provides favorable outcomes for the treatment of chronic ulcers. ${ }^{8}$ Sucralfate's wound protection and repairing mechanism is related to its ability to maintain the integrity of blood vessels. As we have all known, one of the main pathology mechanisms in diabetes mellitus is vasculopathy. Therefore, sucralfate helps the maintenance of proper blood flow; thus improving the bioavailability of wound healing growth factors. ${ }^{10}$

Platelet-rich plasma (PRP) is a preparation made from fresh whole blood which contains more platelet per volume in comparison to the fresh whole blood. One of the most common usages of PRP in the dermatology field is for the treatment of chronic ulcers. ${ }^{11}$ A recent research shows that the application of platelet-rich plasma for the therapy of diabetic ulcers enhanced the wound closure of diabetic ulcers when compared to conventional therapy. ${ }^{9}$

PDGF (Platelet-derived Growth Factor) is a critical growth factor that is involved in wound healing, especially diabetic wounds. The expressions of PDGF and its receptors are decreased in patients with diabetes and diabetic ulcer, therefore an increase of PDGF expression can be used as a marker in diabetic ulcer healing. ${ }^{12}$ Besides PDGF, another growth factor that greatly affecting wound healing and affected by the diabetic condition is
Vascular Endothelial Growth Factor (VEGF). Several researches have shown that VEGF plays an important role in angiogenesis and wound healing. The increase of VEGF expression in the wound bed directly correlates with the rate of regeneration. ${ }^{12-14}$

Hypoxia-inducible factor 1 (HIF-1) is the primary oxygen homeostasis regulator which greatly affects healing outcomes. HIF-1 consists of two subunits, but only HIF$1 \alpha$ is affected by oxygen levels. ${ }^{13,14}$ Patients with diabetes mellitus experienced chronic hypoxic conditions due to hyperglycemia and metabolic stress, which results in increased HIF-1 $\alpha$ expression. ${ }^{15,16}$ Therefore, the expression of HIF-1 $\alpha$ is an important indicator to determine the healing of diabetic ulcers.

One of the main cells that plays a great role in wound healing is the macrophage. It works by secreting growth factors, cytokines (both proinflammatory cytokines and antiinflammatory cytokines), and collagen synthesis. Macrophage actions affect wound tensile strength and helps the re-epithelialization of wounds. In chronic wounds, such as diabetic ulcers, the capability of macrophage to phagocyte neutrophils is severely diminished, and macrophage may increase the inflammation in the wound area, further disturbing the wound healing process in diabetic wounds. ${ }^{17}$

Both sucralfate and PRP in its monotherapy usage enhances wound healing and shorten the duration of diabetic ulcers wound healing. However, the combination of sucralfate and PRP for the treatment of diabetic ulcers has never been investigated before. Therefore, this study was conducted to evaluate the potential of sucralfate and PRP combination therapy to improve diabetic ulcers wound healing.

\section{Materials and Methods}

\section{Animal Preparation}

This study is based on a true-experimental, post-test only control group design which were conducted in JanuaryMarch 2020. Ninety male Wistar rats, aged 2-3 months with a mean weight of 150 to 200 grams were randomly divided into five study groups. Each group consists of twelve rats. The rats were further divided into two study periods. In the first period (7 days), half of every group population (six rats) rats were terminated for immunohistochemistry examination. In the second period (14 days), the remaining half of the population was terminated for immunohistochemistry examination.

The group consists of four diabetic groups and one non-diabetic group (Table 1). The diabetic groups received different treatment regiments. Of the four diabetic groups, 
Table I Subject Grouping

\begin{tabular}{|l|l|l|}
\hline Abbreviation & Name & Intervention \\
\hline DC & Diabetic control group & $\begin{array}{l}\text { Diabetic ulcer wound mice model, treated with conventional therapy once a day (wound debridement } \\
\text { with } 0.9 \% \text { normal saline, topical 2\% fusidic acid antibiotic for infection management, and sterile gauze } \\
\text { dressing) }\end{array}$ \\
\hline NC & Non-diabetic control group & Non-diabetic control mice with excisional ulcer wound model with no therapy regiment given. \\
\hline TS & $\begin{array}{l}\text { Topical sucralfate } \\
\text { monotherapy }\end{array}$ & Diabetic ulcer wound mice model, treated with topical sucralfate on the wound site once a day \\
\hline TP & $\begin{array}{l}\text { Topical PRP monotherapy } \\
\text { TSP }\end{array}$ & $\begin{array}{l}\text { Diabetic ulcer wound mice model, treated with topical PRP once a week on the wound site on the 7th } \\
\text { and 14th day }\end{array}$ \\
\hline combination therapy & $\begin{array}{l}\text { Diabetic ulcer wound mice model, treated with combination topical sucralfate and topical PRP. Topical } \\
\text { platelet-rich plasma was given on the first day with a one-week interval and sucralfate application was } \\
\text { given from the second day and was continuously given on every day (except on the 7th and 14th day } \\
\text { when PRP was applied to the wounds). }\end{array}$ \\
\hline
\end{tabular}

three of them are treatment groups; the first group received topical sucralfate only (TS), the second group received topical PRP only (TP), and the third group received a combination of topical sucralfate and topical PRP (TSP). The last diabetic group was assigned as the diabetic-control group which received conventional dressing therapy (DC). The remaining non-diabetic group was assigned as a non-diabetic control group (NC) which received no treatment and serves as a negative control:

All of our rats were kept in the Animal Research Laboratory, Faculty of Medicine, Diponegoro University. All rats were kept in animal research-grade cage with a 12 hours light-dark cycle and a constant temperature between $25^{\circ} \mathrm{C}$ and $28^{\circ} \mathrm{C}$. All rats were provided with a sufficient amount of standard rodent diet and drinking water ad libitum.

\section{Diabetes Induction and Monitoring}

Diabetes induction was done by streptozotocin-nicotinamide $(45 \mathrm{mg} / \mathrm{kg})$ injection. We dissolved streptozotocin $(45 \mathrm{mg} / \mathrm{kg})$ in citrate buffer $(0.1 \mathrm{M}, \mathrm{pH} 4.5) .{ }^{18}$ We used nicotinamide $(100 \mathrm{mg} / \mathrm{kg})$ to prevent the damage in pancreatic cells due to streptozotocin. ${ }^{19}$ This solution was injected intraperitoneally into the rats using a sterile syringe and needle (OneMed, Indonesia). We measured the blood glucose level using the POCT technique using the blood sample from the lateral saphenous vein blood three days after streptozotocin-nicotinamide injection. We considered rats with blood glucose levels greater than $250 \mathrm{mg}$ / $\mathrm{dl}$ as diabetic rats and were allotted randomly to the four diabetic groups as mentioned above. ${ }^{19}$ The measurement of the rats' blood glucose levels was done with a glucometer before and after each treatment session to make sure that each rat is still in a diabetic state. In our research protocol, all rats in this research should meet the minimum blood glucose level of $250 \mathrm{mg} / \mathrm{dL}$ during the study period.

\section{Preparation of Platelet-Rich Plasma}

Autologous platelet-rich plasma was obtained from the rats used in this study. We cannulated the rats at the jugular vein using the technique of Harms and Ojeda (1974). ${ }^{20,21}$ Approximately $3.15 \mathrm{~mL}$ of rats blood were collected using a $5 \mathrm{~mL}$ disposable syringe containing $0.35 \mathrm{~mL}$ of $10 \%$ sodium citrate as an anticoagulant. We then carefully removed the intravenous cannula from the jugular vein was removed, and a hemostatic solution was applied locally at the cannulated site. The remaining open wound was then sutured, making sure that the tissues are aligned to obtain a proper approximation for better wound healing. We centrifugated the blood at $160 \mathrm{G}$ for 20 minutes (environmental temperature $22^{\circ} \mathrm{C}$ ). The centrifugation will cause the blood to be separated. The lower fraction contained the red blood cells and the upper turbid, whiteyellowish fraction contained the blood serum. We marked $1.5-2 \mathrm{~cm}$ below the separation line of the two fractions using a fine permanent marker. We pipetted all the centrifuge tube content above this line and transferred it to another $5 \mathrm{~mL}$ vacuum tube. We then draw a line located near the bottom of the tube which indicates $0.35 \mathrm{~mL}$ of liquid size using a fine permanent marker. We then centrifugated the second vacuum tube at $400 \mathrm{G}$ for 15 minutes. This causes the blood to be further divided into two layers: one fraction above the $0.35 \mathrm{~mL}$ line mark, which became the platelet-poor plasma (PPP) with only a minimal amount of platelets, and the other fraction below the $0.35 \mathrm{~mL}$ line mark, which became the platelet-rich plasma (PRP). ${ }^{22}$ We pipetted the upper layer and transferred it 
into another vacuum tube and stored it in the freezer. The remaining liquid on the vacuum centrifuge tube was the platelet-rich plasma. Before each application, we agitated the PRP by using a vortex mixer. ${ }^{21}$

\section{Immunohistochemistry Staining and Examination}

We terminated the rats with a high dose of intraperitoneal ketamine injection ( $80 \mathrm{mg} / \mathrm{kgBW})$, followed by decapitation. We obtained a tissue sample located within $2 \mathrm{~mm}$ of the wound border for further examination. The sample tissue was fixed using 10\% neutral buffer, followed by paraffin blocking. The paraffin block was heated for 20 minutes at $105^{\circ} \mathrm{C}$ in an antigen retrieval buffer. After paraffin blocking, we incubated the samples in a polyclonal antibody solution. We added diaminobenzidine tetrahydrochloride (DAB) to visualize the antibody on the samples, and afterward, we stained the cells using Hematoxylin-Eosin (HE). We carried out the magnification at 1000x (10x ocular lens times 100x objective lens) magnification in one field of view using an electronic light microscope (Olympus, Japan). Antibodies used in this research is obtained from Dako, Glostrup, Germany.

Statistical analysis was done using SPSS for Windows (IBM Corporation, USA). We used the Shapiro-Wilk test for the normality test. Based on the result of the ShapiroWilk test, we analyzed normally-distributed data using one-way ANOVA test followed by Tukey post-hoc test. On the other hand, we analyzed the abnormally distributed data using Kruskall-Wallis, followed by the MannWhitney test. Significance was set at 0.05 .

The animal samples in this study were treated according to the experimental animal treatment protocol. Ethical clearance for this research was obtained from the Medical Health and Research Ethics Commission (KEPK), Faculty of Medicine, Diponegoro University. The ethical clearance number is 10 / EC/H/FK-UNDIP/II/2020. The welfare and treatment of the animals has been done according to the Guide for the Care and Use of Laboratory Animals, Eighth Edition, by National Research Council of the National Academies, Washington, USA.

\section{Results}

\section{Analysis of PDGF Expressions in Wound} Tissue Cells

In both study periods, the lowest PDGF expression was found in the diabetic control group, whereas the highest PDGF expression was found in the topical sucralfate and platelet-rich plasma combination group. We found an enhancement of PDGF levels between the 7-day period and 14-day period, however, there was no significant difference between those two study periods in all groups $(\mathrm{p}$ $>0.05$ ). The increase of PDGF expression compared to both controls was found to be significant in both topical platelet-rich plasma group and topical sucralfate and platelet-rich plasma combination group for both study periods (See Figures 1, 2 and 3). Microscopic image of PDGF expressions in wound tissue cells can be seen in Figure 3.

\section{Analysis of Vascular Endothelial Growth Factor Expressions in Wound Tissue Cells}

In both study periods, the lowest VEGF expression was found in the diabetic control group, whereas the highest VEGF expression was found in the topical sucralfate and platelet-rich plasma combination group. We found an enhancement of VEGF levels between the 7-day period and 14-day period; however, there were no significant differences between those two study periods in most groups ( $p>0.05$ ) except for the NC group. The increase of VEGF expression compared to both controls was found to be significant in both topical platelet-rich plasma group and topical sucralfate and platelet-rich plasma combination group for both study periods (See Figures 1, 2, 4, 5). Microscopic image of VEDGF expressions in wound tissue cells can be seen in Figure 4.

\section{Analysis of HIF-I $\alpha$ Expressions in Wound Tissue Cells}

In both study periods, the lowest HIF- $1 \alpha$ expression was found in the topical sucralfate group, whereas the highest HIF-1 $\alpha$ expression was found in the topical platelet-rich plasma combination group. There was no significant difference in HIF-1 $\alpha$ expression between those two study periods in all groups $(\mathrm{p}>0.05)$. In both study periods, only topical sucralfate group provides the most significant decrease in HIF-1 $\alpha$ expression compared to both controls and the two remaining intervention groups (See Figures 1 and 2). Microscopic image of HIF-1 $\alpha$ expressions in wound tissue cells can be seen in Figure 5.

\section{Analysis of Macrophage Expressions in Wound Tissue Cells}

In both study periods, the lowest macrophage expression was found in the diabetic control group, whereas the 


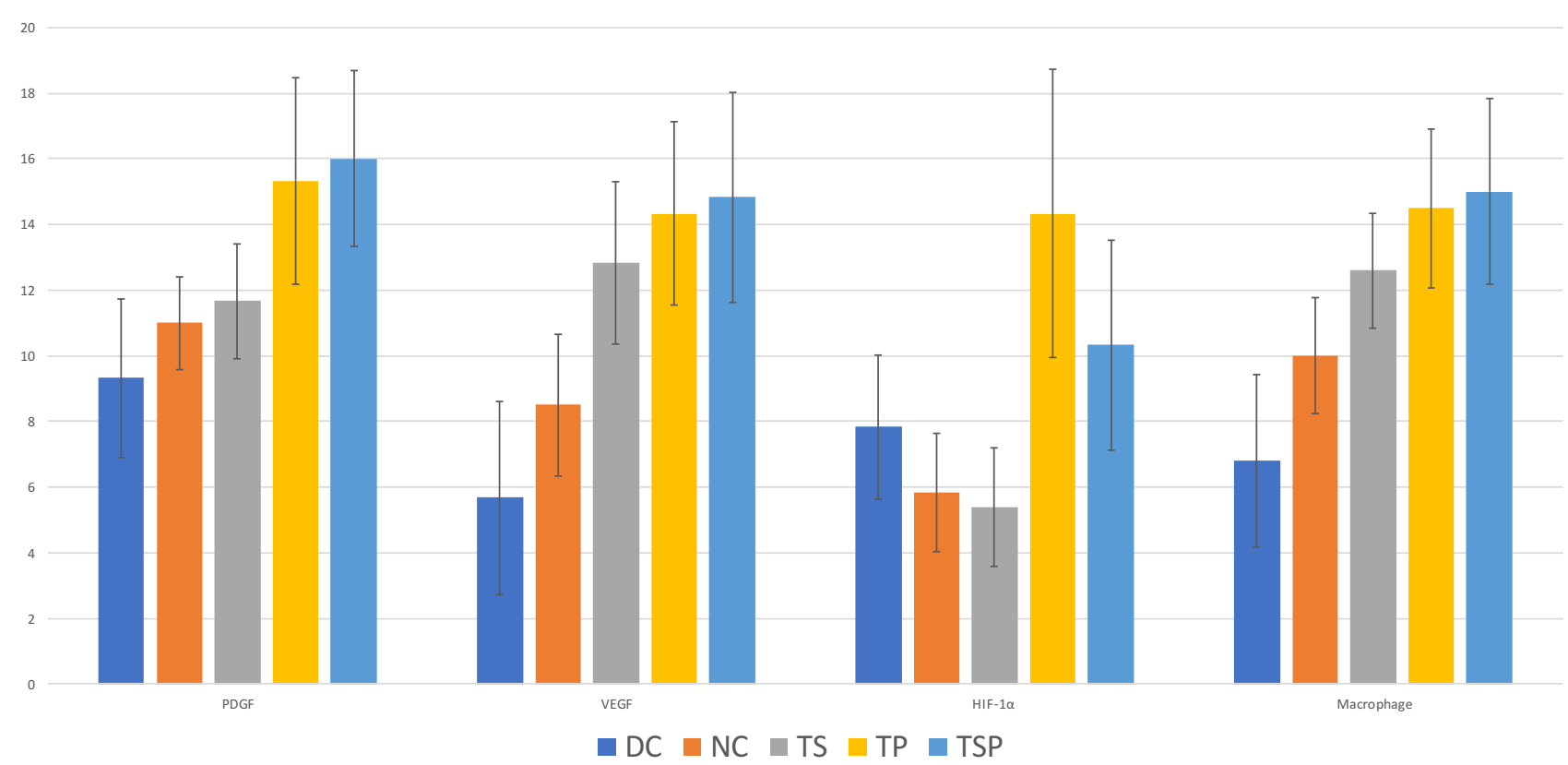

Figure I Comparison of PDGF, VEGF, HIF-I Alpha and Macrophage on 7th Day Study Period.

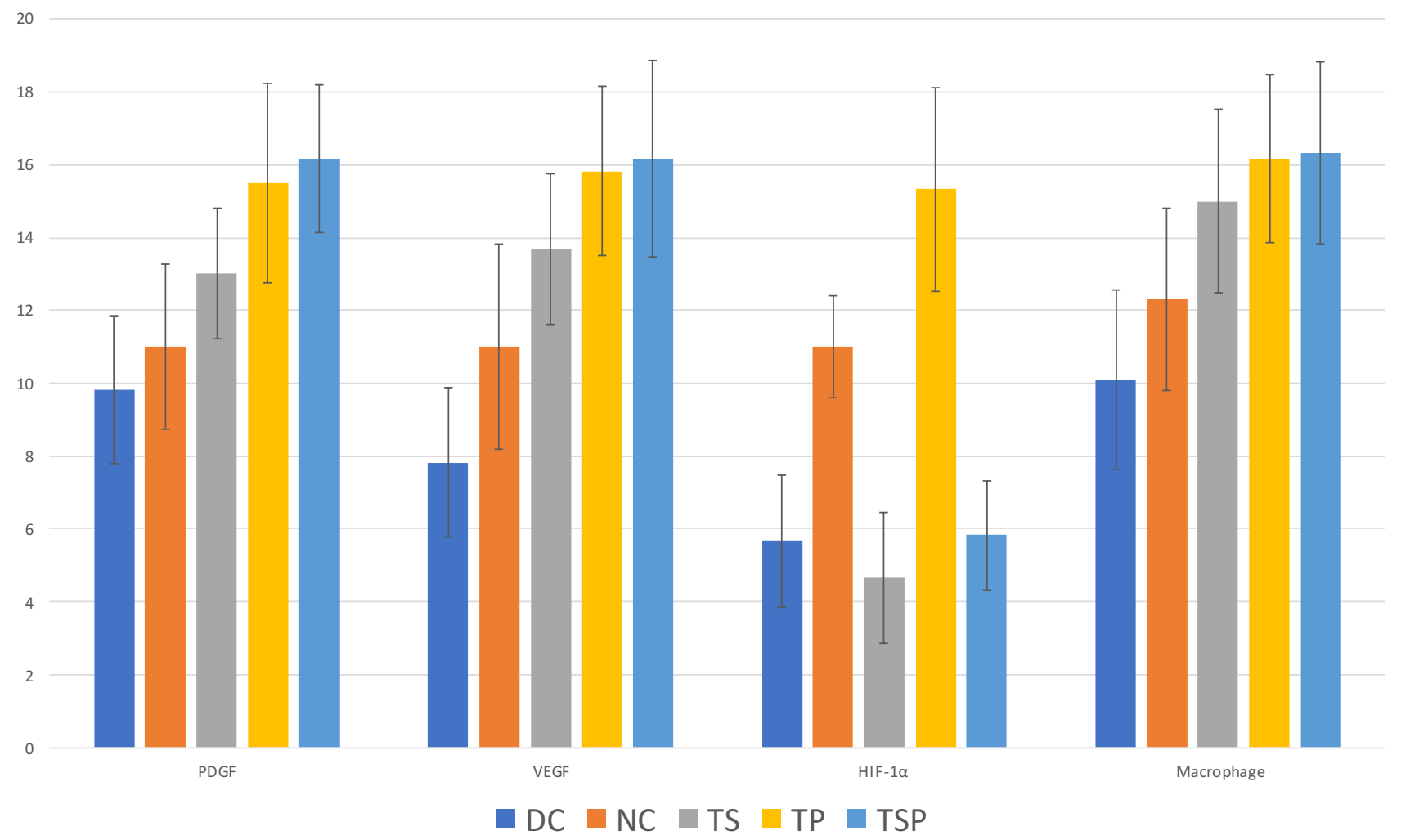

Figure 2 Comparison of PDGF, VEGF, HIF-I Alpha and Macrophage on 14th Day Study Period.

highest macrophage expression was found in the topical sucralfate and platelet-rich plasma combination group. We found an enhancement of macrophage levels between the 7-day period and 14-day period; however, there was no significant difference between those two study periods in all groups $(\mathrm{p}>0.05)$. The increase of macrophage 


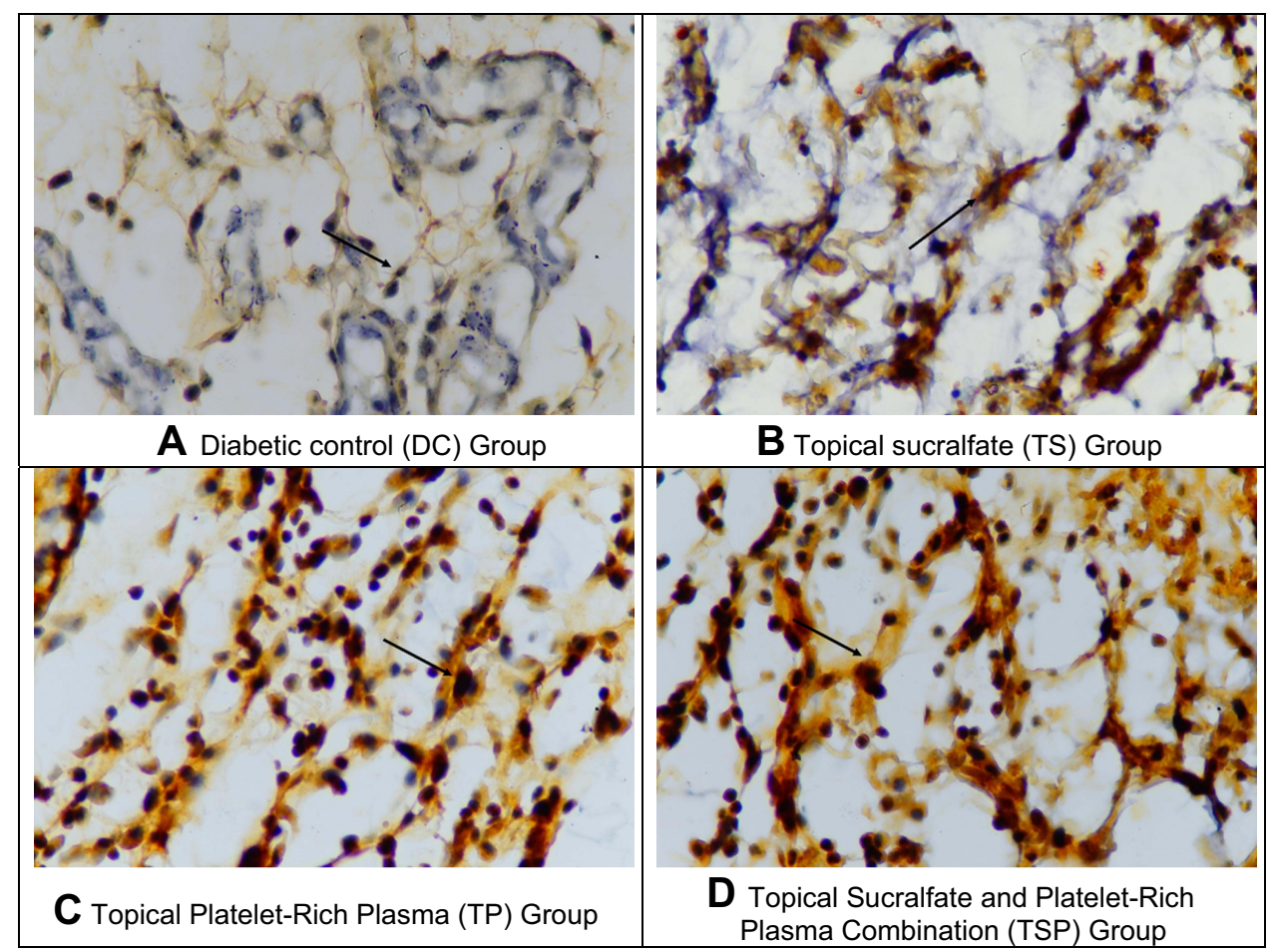

Figure 3 Microscopic image of PDGF expression staining.(A) Diabetic control (DC) group. (B) Topical sucralfate (TS) group. (C) Topical plateletrich plasma (TP) group. (D) Topical sucralfate and platelet-rich plasma combination (TSP) group.

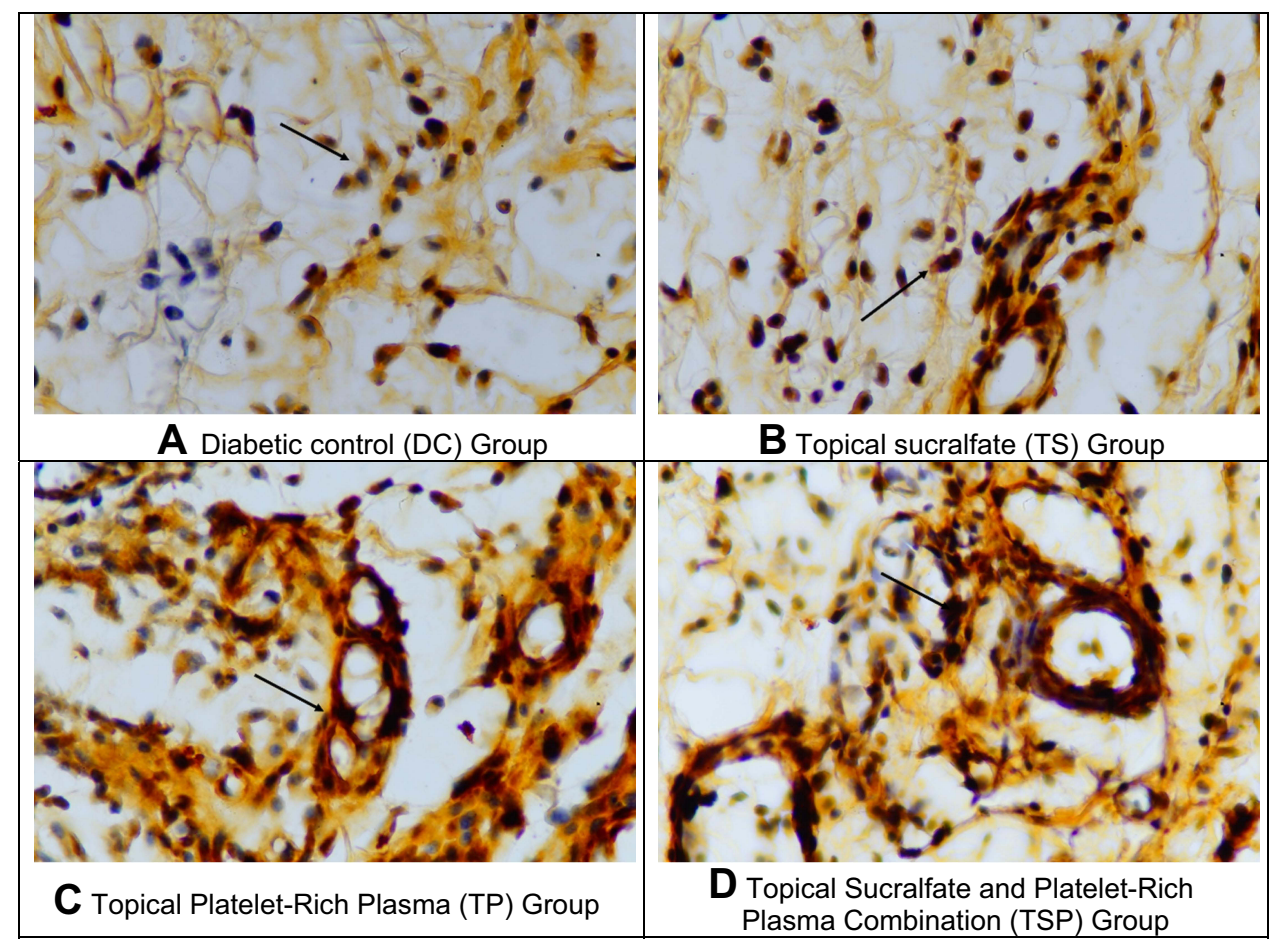

Figure 4 Microscopic image of VEGF expression staining. (A) Diabetic control (DC) group. (B) Topical sucralfate (TS) group. (C) Topical platelet-rich plasma (TP) group. (D) Topical sucralfate and platelet-rich plasma combination (TSP) group. 


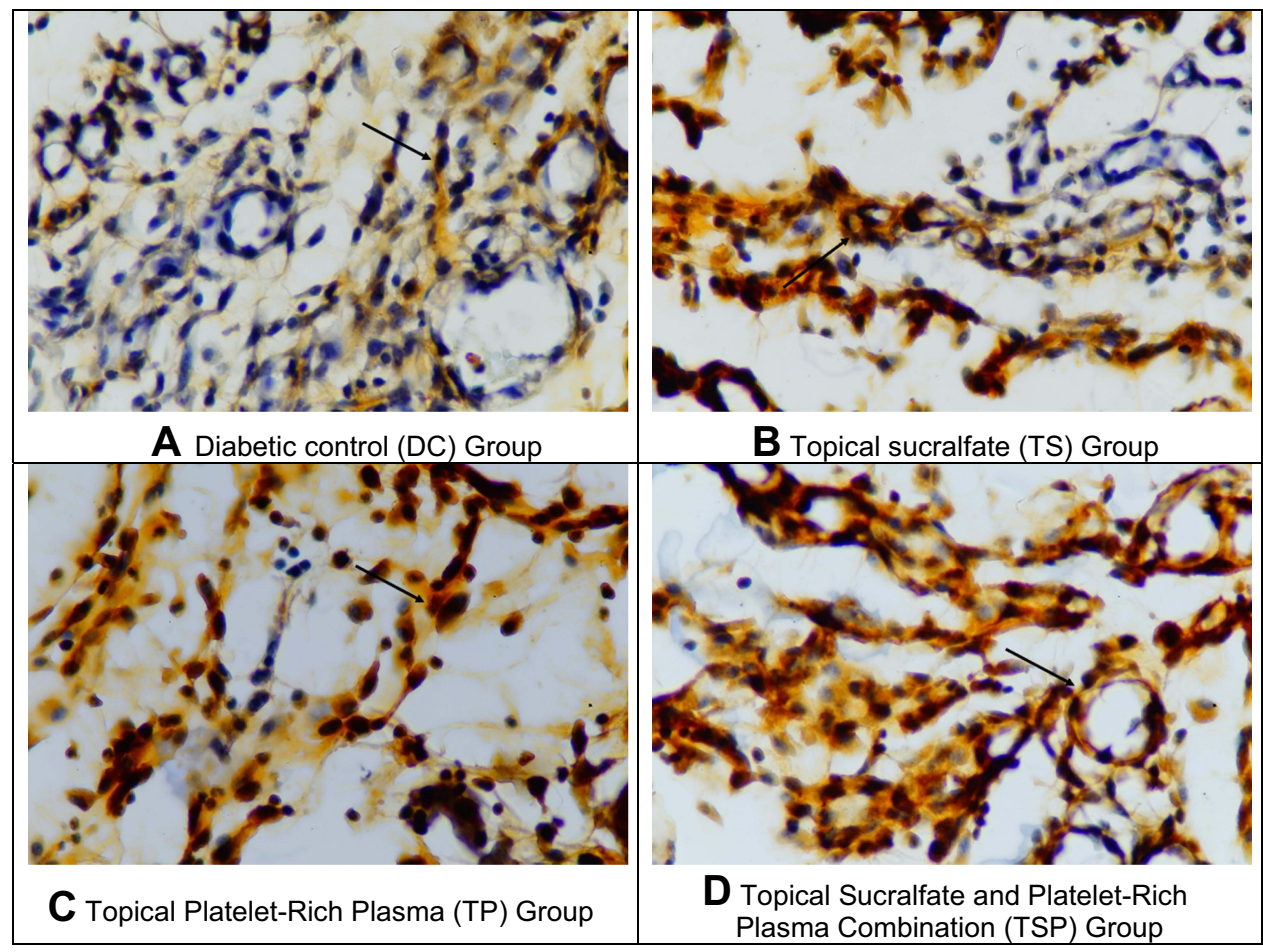

Figure 5 Microscopic image of HIF-I Alpha expression staining. (A) Diabetic control (DC) group. (B) Topical sucralfate (TS) group. (C) Topical plateletrich plasma (TP) group. (D) Topical sucralfate and platelet-rich plasma combination (TSP) group.

expression compared to both controls were found to be significant in both topical platelet-rich plasma group and topical sucralfate and platelet-rich plasma combination group for 7 days study periods. However, the significant increase of macrophage occurs in all study periods in the 14 days study periods but only compared to the diabetic control group (See Figures 1 and 2). Microscopic images of macrophage staining are provided in Figure 6.

\section{Discussion}

The application of sucralfate and PRP combination therapy was found to be most efficacious in this study to accelerate diabetic ulcer healing. There are several mechanisms related to the mechanism of sucralfate and PRP to improve wound healing. Sucralfate decreases reactive oxygen species (ROS) and induces prostaglandin E2 in the inflammatory phase of the wound healing. These mechanisms improve wound protection. The primary sucralfate wound healing mechanism is contributed in the proliferation phase. Sucralfate binds to the specific fibroblast growth factors (bFGF) and epithelial growth factors (EGF), which will result in the acceleration of the neoangiogenesis process and enhance the migration of fibroblasts. ${ }^{23-25}$ The stimulation of bFGF will increase angiogenesis in the wound area. ${ }^{10}$ Besides increasing the angiogenesis, bFGF also acts as a chemoattractive agent and mitogenic factor for several cells, including fibroblasts, endothelial cells, and other mesenchymal cells. These cells contributed to wound contraction and epithelization. The binding of sucralfate to EGF and its receptors will stimulate the keratinocytes migration and proliferation and further improves the reepithelization process. $^{23-25}$

On the other hand, platelet-rich plasma, harnessed by its abundance of growth factors, will release those growth factors contained platelet granules to enhance wound healing. Three PDGF isomers $(\alpha \alpha, \beta \beta$, dan $\alpha \beta)$, two transforming growth factor-beta (TGF- $\beta$ ) isomers ( $\beta 1$ dan $\beta 2$ ), vascular endothelial growth factor (VEGF), and EGF will be released by the platelet-rich plasma. ${ }^{26}$ These growth factors have important roles in every wound healing phases. Platelet-rich plasma also regulates the metalloproteinase matrix (MMP) levels which are needed for the healing of diabetic ulcers. ${ }^{26}$

On days 7 and 14 after the first stage of research, the combination of sucralfate and PRP therapy showed a significantly higher number of cells expressing PDGF compared to conventional therapy and sucralfate monotherapy. The use of this combination therapy in diabetic ulcers also gives significantly higher PDGF expressions than in normal wound healing. These results indicate successful 


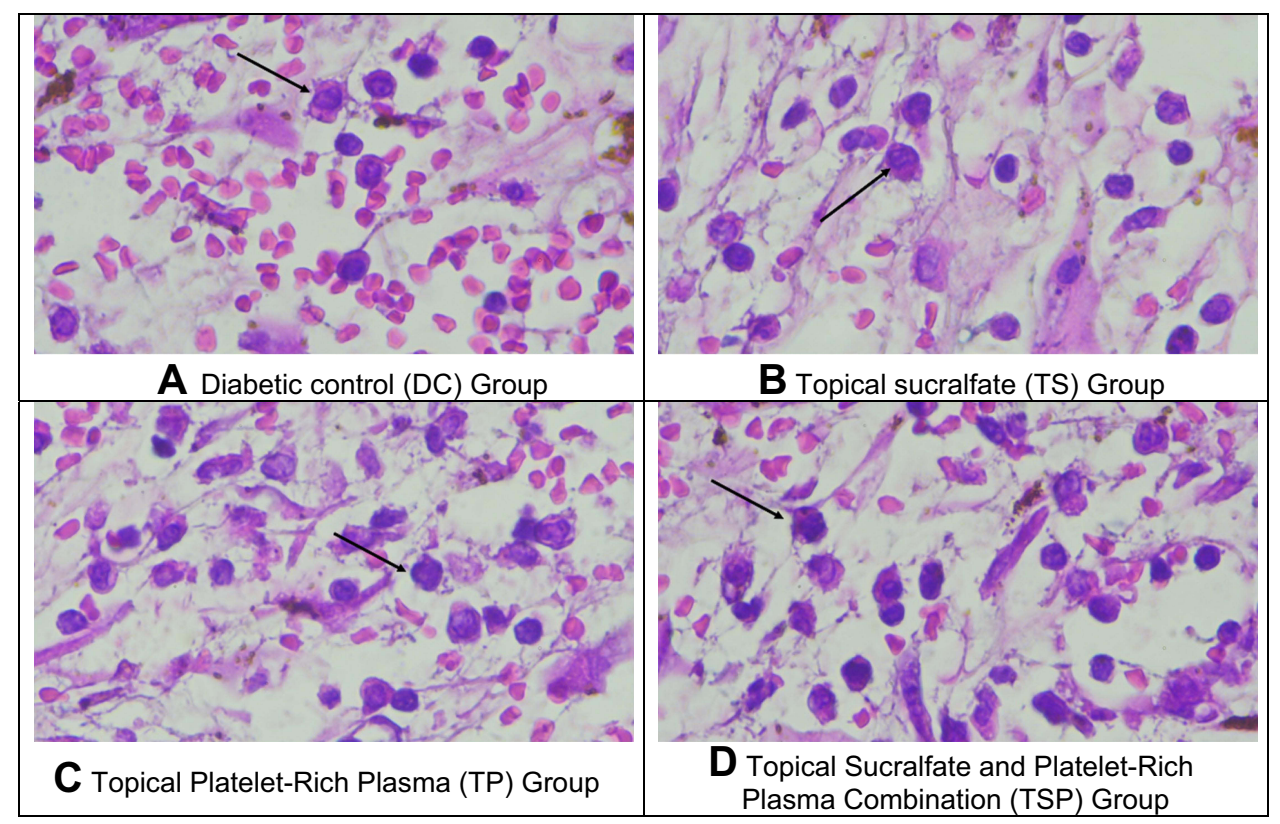

Figure 6 Microscopic image of macrophage staining. (A) Diabetic control (DC) group. (B) Topical sucralfate (TS) group. (C) Topical plateletrich plasma (TP) group. (D) Topical sucralfate and platelet-rich plasma combination (TSP) group.

microscopic healing in diabetic ulcers treated with sucralfate and PRP combination. We speculate that the results came from the synergize effect of combined sucralfate plus PRP.

PRP monotherapy showed a significantly higher number of cells expressing PDGF compared to conventional therapy after 7 and 14 days of treatment. These results correlated with some previous studies about the correlation between PRP therapy and PDGF in wound healing. A study conducted by El-Edel et al showed a significant increase in PDGF concentrations in diabetic ulcer healing treated with PRP gel after 2 weeks. ${ }^{27}$ Another study conducted by Sundman et al showed that PRP therapy increases PDGF and other growth factors in the acceleration of wound healing. ${ }^{28}$ PRP directly increases PDGF expressions in wound tissue cells by releasing PDGF contained in its platelet granules. On the contrary, sucralfate monotherapy did not show a significantly higher number of cells expressing PDGF compared to conventional therapy after 7 and 14 days of treatment. Sucralfate monotherapy also did not show higher PDGF expressions than in normal wound healing. These results are presumably due to an unclear mechanism of sucralfate action in increasing PDGF expression. The main growth factors that bind to sucralfate are FGF and EGF. Sucralfate does not significantly affect PDGF expression in the wound area. ${ }^{10}$ Previous studies showed that sucralfate increased the activation of PDGF receptors to improve the epithelial proliferation in gastric ulcer healing, but there is no further research regarding the role of sucralfate on PDGF in diabetic ulcer healing. ${ }^{29,30}$

In this study, we also found that sucralfate and PRP combination increases the expression of vascular endothelial growth factor in diabetic wound healing. PRP has a direct relationship to increased VEGF expression, which is mediated through platelet degranulation and subsequent release in numerous growth factors that support wound healing. Activated platelets will degranulate and release several mitogenic and chemotactic factors that are important for wound healing and endothelial proliferation, such as VEGF, PDGF, serotonin, histamine, and hydrolytic enzymes. VEGF in diabetic patients is responsible for increasing capillary thickness, tissue perfusion, and metabolism of the ulcer tissue. ${ }^{31}$ The role of PRP in the treatment of diabetic ulcers has also been proven by a metaanalysis study conducted by Del Pino-Sedeño et al. ${ }^{32}$ Platelets contained in PRP can function as an initiator of tissue repair through the release of local growth factors by degranulation of $\alpha$-granules from platelets. The $\alpha$-granule in platelets has a plethora of growth factors, including PDGF and VEGF. ${ }^{33,34}$ Sucralfate has a major role in granulation tissue formation, reepithelization, and fibroblast stimulation in ulcer tissue. ${ }^{10}$ Fibroblast cells secrete prostaglandin E2 in wound tissue, which has a vasodilating effect and also increases VEGF expression. ${ }^{35}$ 
In this study, we found that sucralfate and PRP combination decreases the expression of HIF- $1 \alpha$ in diabetic wound healing. The expression of HIF- $1 \alpha$ in combination therapy was even lower than the expression of HIF- $1 \alpha$ in non-diabetic wound healing. This proves that the therapy of sucralfate, PRP, and the combination of sucralfate and PRP have a positive effect in restoring oxygenation homeostasis of diabetic ulcer tissue. Currently, no theory directly explains the relationship between the sucralfate and PRP mechanisms with HIF- $1 \alpha$ expression. Still, it should be noted that wound healing requires the restoration of the normoxic state through the repair of local microvasculature. ${ }^{36}$ Thus, it is speculated that this positive effect is due to the sucralfate mechanism that increases growth factors such as VEGF and PDGF which can potentiate angiogenesis, ${ }^{10}$ and PRP that contains various growth factors such as PDGF and VEGF which modulate angiogenesis ${ }^{37}$ and result in wound healing.

Topical PRP and sucralfate combination therapy were found to increase macrophage expression in the wound area. This result is similar to the earlier findings by Szalenyi, where the authors stated that sucralfate induces prostaglandin E2, one of the mediators that is synthesized by macrophages and improves wound re-epithelialization and prevents cell apoptosis. ${ }^{38}$ Increased macrophages also alters the wound inflammatory response to favor the tissue remodelling and healing. ${ }^{39}$ Platelet-rich plasma also increases macrophage expression by acting as a chemoattractive agent which increases macrophage migration and activity to the wound area. In the proliferation phase, macrophages will produce PDGF, FGF, and TGF- $\beta$ which play a role in inducing fibroblasts to migrate to the wound area. PDGF and TGF- $\beta$ from PRP have the same role as those produced by macrophages, including attracting fibroblasts to the wound area to further proliferate and produce the ECM matrix. ${ }^{40}$

There are some limitations of this study in terms of determining the actual efficacy of the sucralfate and PRP combination therapy in diabetic patients, due to the difference in the wound area, onset of diabetes, and vascular pathology between humans and diabetic-induced rats. Therefore, we recommend that sucralfate and PRP should be further studied in diabetic patients to evaluate the effectiveness of this combination treatment in diabetic patients. A study using stratified dose topical sucralfate and PRP combination is also needed to correlate and the diabetic ulcers wound healing improvement and different doses of sucralfate and PRP combination.

\section{Conclusion}

Based on the results of this study, it can be concluded that the combination of sucralfate and PRP therapy is the most effective modality to accelerate wound healing in diabetic ulcers after 14 days of treatment compared to the other treatment groups. This combination therapy increases macrophage count, VEGF expression, PDGF expression, and reduces HIF-1 $\alpha$ expression, which will help to increase the effectiveness of the wound healing.

\section{Disclosure}

The authors report no conflicts of interest in this work.

\section{References}

1. Tran TD-X, Le PT-B, Van Pham P. Diabetic foot ulcer treatment by activated platelet rich plasma: a clinical study. Biomed Res Ther. 2014;1(02):37-42. doi:10.7603/s40730-014-0008-3

2. Alexiadou K, Doupis J. Management of diabetic foot ulcers. Diabetes Ther. 2012;3(1):4. doi:10.1007/s13300-012-0004-9

3. Zhang P, Lu J, Jing Y, Tang S, Zhu D, Bi Y. Global epidemiology of diabetic foot ulceration: a systematic review and meta-analysis. Ann Med. 2017;49(2):106-116. doi:10.1080/07853890.2 016.1231932

4. Arosi I, Hiner G, Rajbhandari S. Pathogenesis and treatment of callus in the diabetic foot. Curr Diabetes Rev. 2016;12(3):179-183. doi:10.2174/1573399811666150609160219

5. Yazdanpanah L, Shahbazian H, Nazari I, et al. Risk factors associated with diabetic foot ulcer-free survival in patients with diabetes. Diabetes Metab Syndr. 2018;12(6):1039-1043. doi:10.1016/j. dsx.2018.06.020

6. Alvarsson A, Sandgren B, Wendel C, Alvarsson M, Brismar K. A retrospective analysis of amputation rates in diabetic patients: can lower extremity amputations be further prevented? Cardiovasc Diabetol. 2012;2:11.

7. Sørensen MLB, Jansen RB, Wilbek Fabricius T, Jørgensen B, Svendsen OL. Healing of diabetic foot ulcers in patients treated at the copenhagen wound healing center in 1999/2000 and in 2011/ 2012. J Diabetes Res. 2019. doi:10.1155/2019/6429575

8. Nagalakshmi G, Amalan AJ, Anandan H. Clinical study of comparison between efficacy of topical sucralfate and conventional dressing in the management of diabetic ulcer. Int J Sci Study. 2017;236(3):236.

9. Velayutham S, Velayutham P. A comparative study on the efficacy of platelet rich plasma vs conventional wound dressing in diabetic foot ulcers. Int Surg J. 2019;6(5):1-4. doi:10.18203/2349-2902. isj20191484

10. Masuelli L, Tumino G, Turriziani M, Modesti A, Bei R. Topical use of sucralfate in epithelial wound healing: clinical evidence and molecular mechanisms of action. Recent Pat Inflamm Allergy Drug Discov. 2010;4(1):25-36. doi:10.2174/187221310789895649

11. Hidajat D, Malik DA, Buditjahjono S. Platelet-rich plasma dalam dermatologi. Mdvi. 2012;39(4):176-185.

12. Samuel A, Mahajan A, Mam MK, Prakash JS. Platelet derived growth factor in diabetic lower extremity ulcer: a randomized, double blind, placebo controlled study in Indian condition. Int J Pharm Sci Res. 2016;7(9):3887-3892.

13. Hong WX, Hu MS, Esquivel M, et al. The role of hypoxia-inducible factor in wound healing. Adv Wound Care. 2014;3(5):390-399. doi:10.1089/wound.2013.0520 
14. Biddlestone J, Bandarra D, Rocha S. The role of hypoxia in inflammatory disease (Review). Int j Mol Med. 2015;35(4):859-869. doi:10.3892/ijmm.2015.2079

15. Sada K, Nishikawa T, Kukidome D, et al. Hyperglycemia induces cellular hypoxia through production of mitochondrial ROS followed by suppression of aquaporin-1. PLoS One. 2016;11(7):1-16. doi:10.1371/journal.pone.0158619

16. Cerychova R, Pavlinkova G. HIF-1, metabolism, and diabetes in the embryonic and adult heart. Front Endocrinol. 2018;9(AUG):1-14. doi:10.3389/fendo.2018.00460

17. Krzyszczyk P, Schloss R, Palmer A, Berthiaume F. The role of macrophages in acute and chronic wound healing and interventions to promote pro-wound healing phenotypes. Front Physiol. 2014;3:419. doi:10.3389/fphys.2018.00419

18. Motyl K, McCabe LR. Streptozotocin, type i diabetes severity and bone. Biol Proced Online. 2009;11(1):296-315. doi:10.1007/s12575009-9000-5

19. Ghasemi A, Khalifi S, Jedi S. Streptozotocin-nicotinamide-induced rat model of type 2 diabetes (review). Acta Physiol Hung. 2014;101 (4):408-420. doi:10.1556/APhysiol.101.2014.4.2

20. Harms PG, Ojeda SR. A rapid and simple procedure for chronic cannulation of the rat jugular vein. J Appl Physiol. 1974;36(3):391392. doi:10.1152/jappl.1974.36.3.391

21. Messora MR, Nagata MJH, Furlaneto FAC, et al. A standardized research protocol for platelet-rich plasma (PRP) preparation in rats. RSBO. 2011;8(3):299-304.

22. Messora MR, Nagata MJ, Furlaneto FA, et al. Análise da eficiência do protocolo de dupla centrifugação para o preparo do plasma rico em plaquetas (PRP) - estudo experimental em coelhos. Rev Sul Bras Odontol. 2009;6:291-296.

23. Liu ZJ, Velazquez OC. Angiogenesis in wound healing. In: Encyclopedia of the Eye. Editor: Darlene A. Dartt. 2010:219-229.

24. Gonzalez AC, Costa TF, de Araújo Andrade Z, Medrado AR. Wound healing - a literature review. An Bras Dermatol. 2016;91(5):614-620. doi:10.1590/abd1806-4841.20164741

25. Werner S, Grose R. Regulation of wound healing by growth factors and cytokines. Physiol Rev. 2003;83(3):835-870. doi:10.1152/ physrev.2003.83.3.835

26. Sundman EA, Cole BJ, Karas V, et al. The anti-inflammatory and matrix restorative mechanisms of platelet-rich plasma in osteoarthritis. Am J Sports Med. 2014;376:143-152.

27. El-Edel R, Noreldin R, Basiony A, El-Khateep Y. Platelet-rich plasma in lower limb ulcers. Menoufia Med J. 2019;32(1):261-266.

28. Sundman EA, Cole BJ, Fortier LA. Growth factor and catabolic cytokine concentrations are influenced by the cellular composition of platelet-rich plasma. Am J Sports Med. 2011;39(10):2135-2140. doi:10.1177/0363546511417792
29. Slomiany BL, Liu J, Keogh JP, Piotrowski J, Slomiany A. Enhancement of gastric mucosal epidermal growth factor and platelet-derived growth factor receptor expression by sucralfate. Gen Pharmacol. 1992;23(4):715-718. doi:10.1016/0306-3623(92) 90154-C

30. Konturek SJ, Konturek JW, Brzozowski T, Slomiany BL, Slomiany A. Effects of sucralfate on growth factor availability. Sucralfate. 1995;175-189.

31. Patel S, Srivastava S, Singh MR, Singh D. Mechanistic insight into diabetic wounds: pathogenesis, molecular targets and treatment strategies to pace wound healing. Biomed Pharmacother. 2019;112:108615. Elsevier Masson SAS. doi:10.1016/j. biopha.2019.108615

32. Del Pino-Sedeño T, Trujillo-Martín MM, Andia I, et al. Platelet-rich plasma for the treatment of diabetic foot ulcers: a meta-analysis. Wound Repair Regen. 2019;27:170-182. Blackwell Publishing Inc.. doi:10.1111/wrr. 12690

33. Lacci KM, Dardik A. Platelet-rich plasma: support for its use in wound healing. Yale J Biol Med. 2010;83(1):1-9.

34. El-Sharkawy H, Kantarci A, Deady J, et al. Platelet-rich plasma: growth factors and pro- and anti-inflammatory properties. $J$ Periodontol. 2007;78(4):661-669. doi:10.1902/jop.2007.060302

35. Hatazawa R, Tanaka A, Tanigami M, et al. Cyclooxygenase-2/prostaglandin E 2 accelerates the healing of gastric ulcers via EP 4 receptors. Am J Physiol Gastrointest Liver Physiol. 2007;293(4): G788-G797. doi:10.1152/ajpgi.00131.2007

36. Eisenbud DE. Oxygen in wound healing. nutrient, antibiotic, signaling molecule, and therapeutic agent. Clin Plast Surg. 2012;39 (3):293-310. doi:10.1016/j.cps.2012.05.001

37. Jain NK, Gulati M. Platelet-rich plasma: a healing virtuoso. Blood Res. 2016;51(1):3-5. doi:10.5045/br.2016.51.1.3

38. Szelenyi I, Lanz R. Release of cytoprotective PGE2 from cultured macrophages induced by antacids and sucralfate. Agents Actions. 1986;18(3-4):375-380. doi:10.1007/BF01965000

39. Konop M, Damps T, Misicka A, Rudnicka L. Certain aspects of silver and silver nanoparticles in wound care: a minireview. J Nanomater. 2016;2016:1-10. doi:10.1155/2016/7614753

40. Singh V, Barbosa FL, Torricelli AAM, Santhiago MR, Wilson SE. Transforming growth factor $\beta$ and platelet-derived growth factor modulation of myofibroblast development from corneal fibroblasts invitro. Exp Eye Res. 2014;120:152-160. doi:10.1016/j. exer.2014.01.003
Journal of Experimental Pharmacology

\section{Publish your work in this journal}

The Journal of Experimental Pharmacology is an international, peerreviewed, open access journal publishing original research, reports, reviews and commentaries on all areas of laboratory and experimental pharmacology. The manuscript management system is completely online and includes a very quick and fair peer-review system. Visit http://www.dovepress.com/testimonials.php to read real quotes from published authors. 